\title{
Exploração de fatores de risco de lesões desportivas entre universitários de educação física: estudo a partir de estudantes de Sorocaba/SP*
}

\author{
Marcelo Conte, Edgard Matiello Júnior, Liciana Vaz Arruda Silveira Chalita e \\ Aguinaldo Gonçalves
}

Grupo de Saúde Coletiva/Epidemiologia e Atividade Física

Faculdade de Educação Física - Unicamp

\section{RESUMO}

O objetivo do presente estudo foi identificar os fatores de risco presentes na incidência de lesões desportivas (LD), entre 307 alunos da Faculdade de Educação Física da ACM de Sorocaba; especificamente visou-se explorar associações entre LD, modalidade e região corporal afetada. Trata-se de investigação observacional, transversal, considerando como variáveis independentes sexo, idade, índice de massa corporal (IMC), período de curso, fase do curso e modalidade, e, como dependentes, as referências de LD ocorridas no período de duas semanas. Os dados de interesse foram coletados através de inquérito de morbidade referida. No plano analítico, associações foram testadas pelo teste do qui-quadrado e realizou-se análise multivariada segundo ajuste de modelo logístico. Os principais resultados revelaram: 1) predominância significativa de LD nos membros inferiores (MMII); 2) associação entre estiramento e entorse nos MMII; 3) incidência maior de LD no primeiro semestre comparado com o sétimo; e 4) sexo, idade, período e IMC não se configuraram como fator risco para LD.

Palavras-chave: Lesões desportivas. Morbidade referida. Universitários.

Recebido em 15/11/2001

Segunda versão recebida em 1/5/2002

Aceito em 17/5/2002

Endereço para correspondência:

Marcelo Conte

Rua Benedito Wenceslau Mendes, 171, apto 14/A

18052-000 - Sorocaba, SP

E-mail: contemarcelo@bol.com.br

\section{ABSTRACT}

Risk factors of sports injuries among university students of physical education in Sorocaba, SP

This study is aimed at identifying risk factors for sports injuries (SI) in 307 students of a Physical Education School in Sorocaba, SP. Associations were explored between SI, sports modality, and body region affected. Investigation was observational, cross-sectional, independent variables being gender, age, body mass index (BMI), school period, course stage and modality; reports of SI occurring in the last two weeks were taken as dependent variables. SI data were collected by means of a morbidity questionnaire. Profile analysis was performed by $\chi^{2}$ test and multivariate analysis by logistic modeling. Main results indicated: 1) significant predominance of SI in lower limbs; 2) associations between strain and sprain in lower limbs; 3) higher incidence of SI during the first semester of school as compared to the seventh semester, and 4) gender, age and BMI did not show influence as risk factors for SI.

Key words: Sports injuries. Reported morbidity. University students.

\section{INTRODUÇÃO E OBJETIVOS}

Atualmente, as lesões desportivas (LD) têm extrapolado o âmbito das Ciências do Esporte, configurando-se também, devido a sua magnitude, transcendência e vulnerabilidade, como problema de saúde pública ${ }^{1}$. Nesse sentido, considera-se surpreendente a baixa quantidade de informações disponíveis acerca da freqüência das lesões e de outros efeitos adversos da atividade física (AF), entre a população em geral ${ }^{2}$.

Especificamente, para favorecer a compreensão desse fenômeno, tem-se adotado a classificação das lesões em quatro categorias gerais: 1) aguda; 2) crônica; 3) exacer- 
bação aguda de lesão crônica, e 4) subclínica ${ }^{3}$ Nesse cenário, observa-se que muita atenção é dada às lesões agudas, porém $50 \%$ do total de LD são crônicas e, ainda, a recuperação destas é em torno de 54\% mais lenta do que nas imediatas ${ }^{4}$. De fato, independentemente do tipo, as LD contribuem de maneira significativa para o afastamento, tanto de iniciantes quanto de ativos regulares, da prática de $\mathrm{AF}^{5}$.

$\mathrm{Na}$ verdade, embora exista grande apelo para realização de AF visando a promoção da saúde, a população que faz ou pratica algum tipo de exercício físico, seja no sentido competitivo ou recreativo, fica exposta aos acidentes desta prática $^{6}$.

O desenvolvimento de estratégias para prevenção das LD deve requerer, sobretudo, a quantificação sistemática de diagnósticos específicos das lesões, bem como investigação de potenciais fatores de risco, tais como características pessoais, equipamentos e modelos de treinamento ${ }^{7}$.

Nesse sentido, o objetivo geral do presente estudo é identificar os fatores de risco presentes na incidência de LD (decorrentes das aulas de predomínio prático), entre alunos de licenciatura plena em Educação Física. Especificamente, visam-se explorar associações entre LD, modalidade e região corporal afetada.

Justifica-se a preocupação específica de estudar LD na referida população, devido ao fato de não se encontrarem dados disponíveis da manifestação de LD em estudantes de Educação Física, os quais podem ser considerados como potenciais formadores de opinião na área de atividade física e esportes. De fato, após pesquisa em base de dados especializada em investigações na área de saúde (Biblioteca Virtual de Saúde - Bireme), foram encontrados poucos estudos a respeito de LD em universitários de Educação Física; das cinco investigações levantadas, quatro delas estavam relacionadas às LD ocorridas durante a prática da educação física curricular e não de eventos ocorridos no interior das disciplinas do curso de graduação.

\section{MATERIAL E MÉTODO}

\section{Tipificação do estudo e caracterização das variáveis}

A pesquisa configura-se como observacional, do tipo transversal, considerando como variáveis independentes sexo, idade, índice de massa corporal (IMC), período de curso (diurno ou noturno), semestre cursado e modalidade praticada no momento das $\mathrm{LD}$, e, como dependentes, as referências de LD ocorridas no período de duas semanas.

\section{Local e população de estudo}

Identificaram-se como população de estudo os alunos da Faculdade de Educação Física da ACM de Sorocaba, em total de 307 (115 homens e 92 mulheres) dos 450 matriculados, diferença devida à dificuldade em ter acesso a todos (por motivo de provas ou faltas). A população estudada apresentou média de idade e desvio padrão, respectivamente, de $23,48 \pm 4,82$ anos para o sexo masculino e $22,25 \pm$ 3,46 para o feminino.

$\mathrm{Na}$ grade curricular da referida Faculdade, o curso é desenvolvido em oito semestres e todos os acadêmicos são submetidos obrigatoriamente a disciplinas com conteúdos de natureza prática e teórica, destacando-se como as de predomínio aplicado: Atletismo, Handebol e Futebol (presentes nos dois primeiros semestres); Basquetebol, Natação e Dança/Rítmica (no terceiro e quarto); Voleibol (no quinto e sexto) e, ainda, a Ginástica, visando o condicionamento físico, ministrada em todos os semestres ${ }^{8}$.

\section{Delineamento observacional}

Como instrumento de coleta de dados empregou-se o inquérito de morbidade referida, consistindo na aplicação de questionário dirigido, procedimento considerando adequado para investigações populacionais devido, principalmente, a sua aplicabilidade e objetividade ${ }^{9,10}$. Ademais, os questionários de forma geral são freqüentemente utilizados em decorrência do baixo custo, abrangência de informações e conveniência em estudos epidemiológicos ${ }^{11,12}$.

Após ter sido demarcado o início e final da coleta de dados, os pesquisadores visitaram pessoalmente cada turma para informar que, após duas semanas, os alunos seriam questionados a respeito das LD ocorridas exclusivamente durante a prática das modalidades esportivas no curso, enfatizando que ficassem atentos para eventuais acidentes e respectivas consequiências pessoais. Para se considerar LD, foram adotados os critérios adaptados ${ }^{13}$ de ocorrência do agravo suficiente para retirar da prática da aula ou para levar à busca de assistência profissional na área da saúde.

O IMC expresso em $\mathrm{kg} / \mathrm{m}^{2}$ foi obtido a partir de informações de peso e estatura fornecidos pelos próprios universitários, pois a reprodutibilidade de tais medidas é satisfató$\mathrm{ria}^{14}$, em investigações em tal grupo populacional. Nesse sentido, adotou-se a seguinte categorização, proposta pela Organização Mundial da Saúde ${ }^{15}:$ 1) desnutrição: IMC < $18,5 \mathrm{~kg} / \mathrm{m}^{2}$; 2) baixo peso: IMC entre 18,6 e $19,9 \mathrm{~kg} / \mathrm{m}^{2} ; 3$ ) peso adequado: IMC entre 20,0 e $24,9 \mathrm{~kg} / \mathrm{m}^{2} ; 4$ ) sobrepeso: IMC entre 25,0 a $29,9 \mathrm{~kg} / \mathrm{m}^{2}$; e 5) obesidade: IMC > 30,0kg/ $\mathrm{m}^{2}$.

Foram inicialmente informados, a respeito da pesquisa, 380 alunos; no entanto, ao final do período de observação, 73 destes não estavam presentes ou não responderam ao inquérito, sendo, portanto, incluídos 307 (80,78\%) para estudo. 


\section{Procedimentos estatísticos}

Associações foram testadas pelo teste do qui-quadrado ${ }^{16}$ e análise multivariada segundo ajuste de modelo logísti$\mathrm{co}^{17}$, ambas interpretadas no nível de 5\% de significância. Tais resultados são expressos sob a forma de apresentação tabular, de acordo com preconizado pelas normas vigentes ${ }^{18}$.

\section{RESULTADOS}

A tabela 1 apresenta a distribuição das LD segundo variáveis descritivas de interesse, revelando como mais freqüente no plano tegumentar a contusão, no muscular o estiramento e no osteoarticular a entorse; quanto às regiões mais afetadas, destacam-se o joelho, dedos e abdome e, finalmente, a modalidade coletiva de maior incidência foi o futebol e, entre as modalidades individuais, o atletismo.

Na tabela 2 realizou-se análise de associação de LD por plano de ocorrência com modalidade, não se detectando significância estatística; no entanto, ao se comparar segundo segmento, a incidência de LD foi significantemente maior nos membros inferiores (MMII) em cada um dos três planos considerados.

Contudo, estudando as 38 LD mais freqüentes (contusão, estiramento e entorse), segundo disciplina e segmento, obteve-se associação entre estiramento e entorse nos MMII (tabela 3).

A análise multivariada procedida (tabela 4), segundo ajuste do modelo logístico, das variáveis independentes, revela incidência de LD no primeiro semestre maior do que no sétimo, considerado como referência, por justamente conter apenas uma disciplina de natureza aplicada. Contudo, as variáveis sexo, idade, período e IMC não se configuraram como fator risco para acometimento de LD, nesta população estudada.

\section{DISCUSSÃO}

Tais achados são discutidos, respectivamente, na perspectiva da predominância significativa de LD, em todos os planos, nos MMII comparados com os MMSS e com o tronco e ainda, no sentido do questionamento das evidências de que, principalmente, mulheres e pessoas com sobrepeso ou obesas seriam particularmente vulneráveis ao acometimento de LD.

De forma geral, o impacto das LD na saúde pública temse mostrado cada vez mais evidente. Verificou-se, em estudo com 12.403 pacientes de hospital holandês ${ }^{19}$, que as lesões geradas pela prática de esportes eram a segunda maior causa de internação e a terceira na escala de gravidade da lesão, quando comparadas com as que ocorreram no lar ou trabalho, em acidentes de trânsito ou decorrentes da vio- lência. Os principais fatores de risco para esse agravo são lesões preexistentes, queixas agudas, reabilitação inadequada, pobre percepção de saúde, alto nível de estresse

\section{TABELA 1}

Distribuição das LD segundo variáveis descritivas de interesse

\begin{tabular}{|c|c|c|c|}
\hline \multicolumn{2}{|c|}{ Variáveis descritivas de interesse } & \multicolumn{2}{|c|}{ Freqüência+ } \\
\hline Plano & Tipo & Absoluta & Relativa \\
\hline \multirow[t]{3}{*}{ Tegumentar } & & 17 & 37,78 \\
\hline & Contusão & 13 & 28,89 \\
\hline & Abrasão & 4 & 8,89 \\
\hline \multirow[t]{2}{*}{ Muscular } & & 16 & 35,56 \\
\hline & Estiramento & 16 & 35,56 \\
\hline \multirow[t]{4}{*}{ Osteoarticular } & & 12 & 26,66 \\
\hline & Entorse & 9 & 20,00 \\
\hline & Luxação & 2 & 4,44 \\
\hline & Fratura & 1 & 2,22 \\
\hline Segmento & Região & & \\
\hline \multirow[t]{9}{*}{ MMII } & & 33 & 73,32 \\
\hline & Joelho & 7 & 15,56 \\
\hline & Tornozelo & 4 & 8,88 \\
\hline & Perna & 4 & 8,88 \\
\hline & Coxa & 4 & 8,88 \\
\hline & Pé & 3 & 6,67 \\
\hline & Dedos & 3 & 6,67 \\
\hline & Hálux & 2 & 4,44 \\
\hline & Outros & 6 & 13,33 \\
\hline \multirow[t]{6}{*}{ MMSS } & & 7 & 15,56 \\
\hline & Dedos & 2 & 4,44 \\
\hline & Punho & 1 & 2,22 \\
\hline & Mão & 1 & 2,22 \\
\hline & Braço & 1 & 2,22 \\
\hline & Outros & 2 & 4,44 \\
\hline \multirow[t]{3}{*}{ Tronco } & & 5 & 11,12 \\
\hline & Abdome & 4 & 8,90 \\
\hline & Ombro & 1 & 2,22 \\
\hline Modalidade & Disciplina & & \\
\hline \multirow[t]{5}{*}{ Coletiva } & & 27 & 60,00 \\
\hline & Futebol & 15 & 33,33 \\
\hline & Basquete & 6 & 13,33 \\
\hline & Handebol & 4 & 8,90 \\
\hline & Voleibol & 2 & 4,44 \\
\hline \multirow[t]{6}{*}{ Individual } & & 18 & 40,00 \\
\hline & Atletismo & 10 & 22,23 \\
\hline & Ginástica & 5 & 11,11 \\
\hline & Rítmica & 1 & 2,22 \\
\hline & Dança & 1 & 2,22 \\
\hline & Treinamento & 1 & 2,22 \\
\hline Total & & 45 & 100,00 \\
\hline
\end{tabular}


TABELA 2

Análise de associação de LD por plano de ocorrência com segmento e modalidade

\begin{tabular}{|c|c|c|c|c|c|c|c|c|}
\hline \multirow{3}{*}{$\begin{array}{l}\text { Variável de } \\
\text { interesse }\end{array}$} & \multicolumn{6}{|c|}{ Plano } & & \\
\hline & \multicolumn{2}{|c|}{ Tegumentar } & \multicolumn{2}{|c|}{ Muscular } & \multicolumn{2}{|c|}{ Osteoarticular } & \multicolumn{2}{|c|}{ Total } \\
\hline & $\mathbf{N}$ & $\%$ & $\mathbf{N}$ & $\%$ & $\mathbf{N}$ & $\%$ & $\mathbf{N}$ & $\%$ \\
\hline \multicolumn{9}{|l|}{ Segmento } \\
\hline MMII & 11 & 24,44 & 13 & 28,89 & 9 & 19,99 & 33 & 73,32 \\
\hline MMSS & 4 & 8,90 & 1 & 2,22 & 2 & 4,44 & 7 & 15,56 \\
\hline Tronco & 2 & 4,44 & 2 & 4,44 & 1 & 2,22 & 5 & 11,12 \\
\hline Total & 17 & 37,78 & 16 & 35,56 & 12 & 26,66 & 45 & 100,00 \\
\hline Comparação & \multicolumn{2}{|c|}{$\begin{array}{l}\chi^{2}=7,882 \\
(p=0,019)\end{array}$} & \multicolumn{2}{|c|}{$\begin{array}{l}\chi^{2}=16,625 \\
(p=0,001)\end{array}$} & \multicolumn{2}{|c|}{$\begin{array}{l}\chi^{2}=9,500 \\
(p=0,009)\end{array}$} & & \\
\hline \multicolumn{9}{|l|}{ Modalidade } \\
\hline Coletiva & 13 & 28,89 & 7 & 15,56 & 7 & 15,56 & 27 & 60,00 \\
\hline Individual & 4 & 8,89 & 9 & 19,99 & 5 & 11,12 & 18 & 40,00 \\
\hline Total & 17 & 37,78 & 16 & 35,56 & 12 & 26,68 & 45 & 100,00 \\
\hline Comparação & \multicolumn{2}{|c|}{$\begin{array}{c}\chi^{2}=2,882 \\
(p=0,090)\end{array}$} & \multicolumn{2}{|c|}{$\begin{array}{l}\chi^{2}=0,001 \\
(p=0,999)\end{array}$} & \multicolumn{2}{|c|}{$\begin{array}{c}\chi^{2}=0,333 \\
(p=0,564)\end{array}$} & & \\
\hline
\end{tabular}

TABELA 3

Análise de associação das LD mais freqüentes $(n=38)$ segundo Disciplina e segmento

\begin{tabular}{|c|c|c|c|c|c|c|c|c|c|}
\hline \multirow{3}{*}{$\begin{array}{l}\text { Variável de } \\
\text { interesse }\end{array}$} & \multicolumn{9}{|c|}{ LD } \\
\hline & \multicolumn{3}{|c|}{ Contusão } & \multicolumn{3}{|c|}{ Estiramento } & \multicolumn{3}{|c|}{ Entorse } \\
\hline & Categoria & $\mathbf{N}$ & $\%$ & Categoria & $\mathbf{N}$ & $\%$ & Categoria & $\mathbf{N}$ & $\%$ \\
\hline \multirow[t]{4}{*}{ Disciplina } & Futebol & 8 & 61,53 & Atletismo & 6 & 37,50 & Futebol & 2 & 22,22 \\
\hline & Outras & 5 & 38,47 & Futebol & 5 & 31,25 & Basquete & 2 & 22,22 \\
\hline & - & - & - & Outras & 5 & 31,25 & Ginástica & 2 & 22,22 \\
\hline & - & - & - & - & - & - & Outras & 3 & 33,34 \\
\hline Total & & 13 & 100,00 & & 16 & 100,00 & & 9 & 100,00 \\
\hline Comparação & \multicolumn{3}{|c|}{$\chi^{2}=0,692(p=0,465)$} & \multicolumn{3}{|c|}{$\chi^{2}=0,125(p=0,939)$} & \multicolumn{3}{|c|}{$\chi^{2}=0,333(p=0,954)$} \\
\hline \multirow[t]{2}{*}{ Segmento } & MMII & 9 & 69,23 & MMII & 13 & 81,25 & MMII & 8 & 88,88 \\
\hline & Outras & 4 & 30,77 & Outras & 3 & 18,75 & Outras & 1 & 11,12 \\
\hline Total & & 13 & 100,00 & & 16 & 100,00 & & 9 & 100,00 \\
\hline Comparação & \multicolumn{3}{|c|}{$\chi^{2}=1,923(p=0,166)$} & \multicolumn{3}{|c|}{$\chi^{2}=6,250(p=0,012)$} & \multicolumn{3}{|c|}{$\chi^{2}=5,444(p=0,002)$} \\
\hline
\end{tabular}

pessoal, características da modalidade, tempo de reação deficiente, baixa resistência física e preparação insuficiente para a competição ${ }^{20}$. Vale destacar que, segundo estudos epidemiológicos, a condição econômica mais baixa (pelo menos em crianças e jovens) também pode ser importante aspecto a ser considerado nessa interação ${ }^{21}$.

Nesse sentido, embora as lesões cerebrais traumáticas ou concussões apresentem maiores implicações à saúde, principalmente a longo prazo ${ }^{22}$, as LD agudas mais freqüen- tes têm atingindo as extremidades, como mãos, pulsos e joelhos ${ }^{23}$. De fato, como na presente investigação, as LD nos MMII foram também significativamente mais elevadas, em vários estudos observados ${ }^{24,25}$; as possíveis explicações para essa situação apontam para o desequilíbrio de força muscular e a baixa flexibilidade nos músculos isquiostibiais $^{26}$.

Outro aspecto interessante do presente estudo foi que sexo e composição corporal não se manifestaram como 
TABELA 4

Análise multivariada, segundo ajuste do modelo logístico, das variáveis independentes

\section{Variável}

Idade

Masculino

Feminino

Período diurno

Período noturno

7은 Semestre

1을 Semestre

$2^{\circ}$ Semestre

$3^{\circ}$ Semestre

$4^{\circ}$ Semestre

$5^{\circ}$ Semestre

6을 Semestre

Peso adequado

Desnutrição

Baixo peso

Sobrepeso

\section{Estimativa}

$-0,015(p=0,722)$

referência

$0,343(p=0,373)$

referência

$0,574(p=0,113)$

referência

$-1,238(p=0,030) *$

$-0,685(p=0,253)$

$0,174(p=0,796)$

$-0,818(p=0,330)$

$-0,071(p=0,939)$

$0,661(p=0,561)$ referência

$0,030(p=0,966)$

$-0,472(p=0,316)$

$0,636(p=0,233)$
Obs.: 0 ${ }^{\circ}$ semestre não foi considerado na modelagem por não ter havido referência a LD nesses grupos.

fatores de risco, ao contrário do mencionado na literatura corrente, que aponta as mulheres duas vezes mais vulneráveis de se lesionarem do que homens e que pessoas com IMC mais elevado teriam risco aumentado em comparação com as de peso adequado ${ }^{27,28}$.

Contudo, após ampla revisão bibliográfica, tais considerações sustentam-se partindo de princípios anátomo-fisiológicos de que as mulheres seriam mais vulneráveis, devido ao fato de possuírem menor massa muscular e maior ângulo Q em comparação com o sexo masculino ${ }^{28}$. No entanto, na infância, quando as diferenças corporais entre meninos e meninas são menores, aspectos comportamentais parecem determinar a distribuição das lesões, pois a literatura revela que a ocorrência de LD em garotos é maior do que em $\operatorname{garotas}^{29,30}$.

\section{REFERÊNCIAS}

1. Gonçalves A, editor. Saúde coletiva e urgência em educação física. Campinas: Papirus, 1997.

2. Powell KE, Heath GW, Kresnow MJ, Sacks JJ, Branche CM. Injury rates from walking, gardening, weightlifting, outdoor bicycling, and aerobics. Med Sci Sports Exerc 1998;30:1246-9.

3. Kibler WB. Clinical aspects of muscle injury. Med Sci Sports Exerc 1990;22:450-2.

4. DiFiori JP. Overuse injuries in children and adolescents. The Physician and Sportsmedicine 1999;27:75-89.

5. Pollock ML, Carrol JF, Graves JE, Leggett SH, Braith RW, Limacher $\mathrm{M}$, et al. Injuries and adherence to walk/jog and resistance training programs in the elderly. Med Sci Sports Exerc 1991;23:1194-200.
Bastante controversa é a questão, embora não explícita nesta pesquisa, do nível de aptidão física (NAF) na determinação das LD, pois existe a concepção de que pessoas com condicionamento físico baixo estariam sujeitas às LD; no entanto, revelou-se que alta aptidão física e volume de atividade física por semana estariam associadas na determinação de lesões, mencionando que, para a maioria dos adultos, a caminhada seria o tipo de atividade mais segu$\mathrm{ra}^{31}$. Tais considerações sugerem que, além de evidências fisiológicas, aspectos sociais e comportamentais devem ser levados em conta no entendimento das LD, pois, talvez, ativos com bom NAF estariam mais expostos e se arriscariam de forma adicional do que indivíduos iniciantes. Dessa forma, faz-se novamente presente a sensação de pseudo-segurança proporcionada pelo exercício, já discutida em outras oportunidades ${ }^{6}$.

\section{CONCLUSÃO}

A partir dos resultados obtidos, pode-se concluir que, entre os alunos de licenciatura plena em Educação Física investigados, encontrou-se: 1) predominância significativa de LD nos membros inferiores (MMII); 2) associação entre estiramento e entorse nos MMII; 3 ) incidência maior de LD no primeiro semestre comparado com o sétimo, e 4) sexo, idade, período e IMC não se configurando como fator risco para LD.

Finalmente, pode-se registrar que o presente estudo adicionou dados para o entendimento das LD em grupo específico, como o de universitários de Educação Física, por um lado questionando determinados fatores de risco e, por outro, revelando que aspectos sociais e comportamentais também devem ser considerados para compreensão desse importante fenômeno das Ciências do Esporte.

Todos os autores declararam não haver qualquer potencial conflito de interesses referente a este artigo.

6. Conte M. Atividade física, um paradoxo à saúde? Estudo a partir de universitários recém-ingressos ao Curso de Medicina da Faculdade de Ciência Médicas da Unicamp [Dissertação]. Campinas: Fef/Unicamp, 2000 .

7. Almeida SA, Williams KM, Shaffer RA, Brodine SK. Epidemiological patterns of musculoskeletal injuries and physical training. Med Sci Sports Exerc 1999;31:1176-2.

8. Matiello Júnior E, Conte M, Gonçalves A, Tóffoli JR. Lesões desportivas entre universitários de Educação Física: em busca de pistas pedagógicas a partir de estudo de determinantes através de morbidade específica referida em Sorocaba/SP. Revista Paranaense de Educação Física 2000; $1: 33-43$. 
9. Carvalheiro JR. Investigação epidemiológica e entrevistas domiciliares. Rev Saúde Pública 1998;15:543-50.

10. Kroeger A. Health interview surveys in developing countries: a review of the methods and results. Int J Epidemiol 1983;12:465-81.

11. Aaron DJ, Kriska AM, Dearwater SR, Cauley JA, Metz KF, LaPorte RE. Reproducibility and validity of an epidemiology questionnaire to assess past year physical activity in adolescents. Am J Epidemiol 1995; 142:191-8.

12. Raugh MJD, Hovell MF, Hofsetter CR, Sallis JF, Gleghorn A. Reliability and validity of self-reported physical activity in latinos. Int J Epidemiol 1992;21:966-71.

13. Garrick JC. Prevention made at American College of Sports and Medicine Conference, Anahein, 1976. In: Klafs CE, Lyon JM. A mulher atleta: guia de condicionamento e treinamento físico. Rio de Janeiro: Interamericana, 1981.

14. Moraes SA. Reprodutibilidade de medidas antropométricas em alunos de enfermagem de Ribeirão Preto - Brasil. Procedente do 4 Congresso Brasileiro de Epidemiologia; 1998 ago 27 a 30; Rio de Janeiro, Brasil.

15. WHO Expert Committee. Physical status: the use and interpretation of anthopometry. WHO Technical Report Series, n. 854. Geneva: WHO, 1995.

16. Gonçalves A. Os testes de hipóteses como instrumental de validação da interpretação (estatística inferencial). In: Marcondes MA, Lakatos EM. Técnicas em pesquisas. São Paulo: Atlas, 1982.

17. McCullagh P. Regression models for ordinal data (with discussion). Journal the Royal Statistical Society 1980;42:109-42.

18. Padovani CR. Estatística na metodologia da investigação científica. Botucatu: Unesp (Instituto de Biociências), 1995.

19. Dekker R, Kingma J, Groothoff JW, Eisma WH, Ten Duis HJ. Measurement of severity of sports injuries: an epidemiological study. Clin Rehabil 2000;14:651-6.
20. Dvorak J, Junge A, Chomiak J, Graf-Baumann T, Peterson L, Rüsch D, et al. Risk factor analysis for injuries in football players: possibilities for a prevention. Am J Sports Med 2000;28:69-74.

21. Faelker T, Pickett W, Brison RJ. Socioeconomic differences in childhood injury: a population based epidemiologic study in Ontario, Canada. Inj Prev 2000;6:203-8.

22. Bailes JE, Cantu RC. Head injury in athletes. Neurosurgery 2001;48: 26-45.

23. Cohen AR, Metzl JD. Sports-specific concerns in the young athlete: basketball. Pediatr Emerg Care 2000;16:462-8.

24. Murtaugh K. Injury patterns among female field hockey players. Med Sci Sports Exerc 2001;33:201-7.

25. Beekeboom C, Birmingham TB, Forwell L, Ohrling D. Asymmetrical strength changes and injuries in athletes training on a small radius curve indoor track. Clin J Sport Med 2000;10:245-50.

26. Hreljac A, Marshall RN, Hume PA. Evaluation of lower extremity overuse injury potential in runners. Med Sci Sports Exerc 2000;32:1635-41.

27. Knapik JJ, Sharp MA, Chervak-Canham M, Hauret K, Patton JF, Jones B. Risk factors for training-related injuries among men and women in basic combat training. Med Sci Sports Exerc 2001;33:946-54.

28. Pollock ML, Gaesser GA, Butcher JD, Després JP, Dishman RK, Franklin BA, et al. The recommend quantity and quality of exercise for developing and maintaining cardiorespiratory and muscular fitness, and flexibility in healthy adults. Med Sci Sports Exerc 1998;30:971-5.

29. Brudvick C. Child injuries in Bergen, Norway. Injury 2000;31:761-7.

30. Marcenes W, Alessi ON, Traebert J. Causes and prevalence of traumatic injuries to the permanent incisors of school children aged 12 years in Jaraguá do Sul, Brasil. International Dentist Journal 2000;50:87-92.

31. Hootman JM, Macera CA, Ainsworth BE, Martin M, Anddy CL, Blair SN. Association among physical activity level, cardiorespiratory fitness, and risk of musculoskeletal injury. Am J Epidemiol 2001;154:251-8. 